\title{
L-arginina aumenta a produção endotelial de óxido nítrico e reduz a pressão arterial de repouso sem alterar as respostas pressóricas do exercício
}

\author{
L-arginine increases endothelial nitric oxide production and reduces \\ blood pressure of rest without changing the exercise pressor response
}

\author{
J.M. Lima, A.S. Silva, N.F. Alves, S.K. Porpino, A.E. Almeida, R.T. Lima
}

ARTIGO ORIGINAL | ORIGINAL ARTICLE

\begin{abstract}
RESUMO
A suplementação de L-arginina tem sido proposta para melhorar a função endotelial. Testou-se a hipótese de que a L-arginina melhora também a hipotensão pós-exercício (HPE) em hipertensos. Hipertensas fisicamente ativas foram distribuídas em grupo suplementado (SUP) ( $\mathrm{N}=10,50 \pm 1.8$ anos) e placebo (PLA) $(\mathrm{N}=10,51.5 \pm 1.6$ anos). Elas realizaram duas sessões de caminhada em intensidade moderada com intervalo de 32 dias entre as sessões. Coletas sanguíneas e pressão arterial foram medidas pré e pós exercícios. No período entre as sessões, permaneceram fisicamente ativas e receberam 6gr diárias de L-arginina ou placebo. PLA não apresentou alteração na concentração sérica de nitrito/nitrato e PA em repouso ou em resposta ao exercício. SUP apresentou aumento na concentração basal de nitrito/ nitrato plasmático $(26.6 \pm 2$ para $44.6 \pm 4 \mu \mathrm{M}, p<.05)$, que foi acompanhado por redução da PA basal $(137 / 86.2$ para $125.8 / 79 \mathrm{mmHg}, p<.05)$. O exercício realizado antes da suplementação não alterou a concentração sérica de nitrito/nitrato, mas a suplementação fez com que o exercício aumentasse estes níveis de $44.6 \pm 4$ para $51.8 \pm 6 \mu \mathrm{M}(p<.05)$. Entretanto, não promoveu alterações na HPE. A suplementação de L-arginina melhora a produção de nitrito/nitrato e promove redução da PA de repouso, mas não altera a HPE em mulheres hipertensas de meia idade.
\end{abstract}

Palavras-chave: suplementação, pressão arterial, arginina, óxido nítrico, exercício físico

ABSTRACT

L-arginine supplementation has been proposed to improve endothelial. The hypothesis that L-arginine also improves post-exercise hypotension (PEH) in hypertensive individuals was tested in this study. Physically active hypertensive women were divided into supplemented (SUP) $(n=12,50 \pm 1.8$ years) and placebo (PLA) $(n=14,51.5 \pm 1.6$ years) groups performing two walking sessions at moderate intensity with interval of 32 days between sessions. Blood samples were collected and blood pressure (BP) was measured before and after exercise. In the period between sessions, they remained physically active and received daily $6 \mathrm{~g}$ of L-arginine or placebo. PLA showed no change in serum nitrite/nitrate concentration and blood pressure at rest or in response to exercise. SUP showed increased basal nitrite/ nitrate plasma concentration $(26.6 \pm 2$ to $44.6 \pm 4 \mu \mathrm{M}, p<.05)$, which was followed by reduction of basal BP $(137 / 86.2$ to $125.8 / 79 \mathrm{mmHg}, p<.05)$. The exercise performed before supplementation did not change the serum nitrite/nitrate concentration. After supplementation, the exercise promoted increases in levels from $44.6 \pm 4$ to $51.8 \pm 6 \mu \mathrm{M}(p<.05)$. However, it did not change the PEH. L-arginine supplementation improves the nitrite/nitrate production and decreases the resting BP, but does not change the PEH in hypertensive middle-aged women.

Keywords: supplementation, blood pressure, arginine, nitric oxide, physical exercise

Submetido: 30.04.2011 | Aceite: 07.09.2012

Janaína Mota de Lima, Alexandre Sérgio Silva, Antônio Eduardo Monteiro de Almeida e Roberto Teixeira Lima. Departamento de Nutrição.

Naiane Ferraz Bandeira Alves, Suênia Karla Pacheco Porpino. Departameto de Educação Física. Universidade Federal da Paraíba, João Pessoa-PB, Brasil.

Endereço para correspondência: Alexandre Sérgio Silva, R. Monteiro Lobato, 501 / 408 - Tambaú - João Pessoa-PB, Brasil CEP.: 58039-170

E-mail: ass974@yahoo.com.br 
A hipertensão arterial sistêmica (HAS) é a mais prevalente de todas as doenças cardiovasculares. Atualmente atinge cerca de $20 \%$ das populações dos países desenvolvidos e está previsto que em 2025 , cerca de $29 \%$ da população adulta do mundo terá hipertensão (Sarri, Linardakis, Codrington, \& Kafatos, 2007; Sociedade Brasileira de Cardiologia, Sociedade Brasileira de Hipertensão, \& Sociedade Brasileira de Nefrologia, 2006).

Os mecanismos envolvidos na hipertensão arterial ainda não estão completamente elucidados. Até o momento, sabe-se que fatores neurais e humorais estão alterados em sujeitos portadores de hipertensão. Observa-se um desequilíbrio entre os sistemas simpático e parassimpático, com maior estimulação simpática e menor atividade parassimpática (Dequattro \& Feng, 2002; Piccirilo, Munizzi, Fimognari, \& Marigliano, 1996). Além disso, a sensibilidade barorreflexa está diminuída em hipertensos (Accorsi-Mendonça, Almado, Fernandes, \& Machado, 2005; Consolim-Colombo \& Fiorino, 2005; Irigoyen, Lacchini, Angelis, \& Michelini, 2003). Sabe-se que o endotélio exerce importante função na contratilidade e no relaxamento vascular (Vanhoutte, 2003). A função endotelial é reduzida comumente no envelhecimento, como resultado do estresse oxidativo ou ação de lipoproteínas de baixa densidade sobre os vasos (Higashi, Oshima, Ozono, Matsuura, \& Kajiyama, 1997; Jonh \& Schmieder, 2003; Sposito, 2004; Touyz, 2005). Portadores de hipertensão costumam apresentar uma menor produção ou biodisponibilidade de óxido nítrico, o que colabora para promover uma maior vasoconstrição nestes sujeitos (Higashi et al., 1997).

O óxido nítrico exerce ação pleiotrópica no organismo, sendo importante no controle da agregação plaquetária, resistência e crescimento vascular, no ritmo de filtração glomerular e processos inflamatórios, dentre outros fatores (Maeda et al., 2001; Wang, 2005). Nas células endoteliais, o L-arginina, que é um aminoá- cido essencial e sofre a ação de uma enzima produzida no endotélio, a óxido nítrico sintase (eNOS) (Green, Maiorana, O’Driscoll, \& Taylor, 2004; Lassègue \& Clempus, 2003: Tatchum-Talom, Schulz, Mcneill, \& Khadour, 2000). O aumento do fluxo sanguíneo decorrente da prática de exercício físico exerce uma ação denominada estresse de cisalhamento sobre os vasos (Kuru et al., 2002). Mecanoceptores localizados na superfície dos vasos são estimulados pelo estresse de cisalhamento, e dão origem a uma cascata de reações que resultam na estimulação da eNOS, que, por sua vez, utiliza o grupo nitrogênio guanidina da L-arginina para formar óxido nítrico + citrulina (Boo \& Jô, 2003; Danson \& Paterson, 2005; Moncada, Palmer, \& Higgs, 1991). Este é um dos mecanismos pelos quais o exercício físico contribui para a redução da pressão arterial e melhoria da função endotelial em hipertensos (Claudino et al., 2004; Griffin, Laughlin, \& Parker, 1999).

A redução da pressão arterial (PA) com exercícios equivale ao uso de uma das classes de medicação anti-hipertensiva (Baster \& Baster-Brooks, 2005). A variação desta redução é de 2 a $17 \mathrm{mmHg}$ para pressão arterial sistólica (PAS) e 2 a $7 \mathrm{mmHg}$ para pressão arterial diastólica (PAD) (Araújo, 2001; Moncada, 1997). Apenas uma única sessão de exercício já é capaz de promover redução da pressão arterial. Além disso, esta hipotensão se sustenta por 22-24 ou 48 horas após uma sessão de exercício, o que confere relevância clínica para redução aguda da pressão arterial (Araújo, 2001; Baster \& Baster-Brooks, 2005). Esta redução aguda da PA tem sido denominada hipotensão pós-exercício (HPE) (Rebelo, Benetti, Lemos, \& Carvalho, 2001).

Uma vez que a L-arginina é o substrato para a produção do óxido nítrico, estudos têm testado a hipótese de que a suplementação deste aminoácido poderia melhorar a função endotelial (Moncada, 1997). Vários destes estudos têm associado à suplementação de L-arginina com redução da pressão arterial (Martina et al., 
2008; Palloshi et al., 2004; Rytlewski, Olszanecki, Korbut, \& Zdebski, 2005). Além disso, outros benefícios, como melhoria do fluxo sanguíneo e redução da agregação plaquetária, têm sido atribuídos à suplementação de L-arginina em modelos animais e humanos (Adams, Forsyth, Jessup, Robinson, \& Celermajer, 1995; Hambrecht et al., 2000; Lekakis et al., 2002).

Considerando que o efeito vasorrelaxante decorrente do fenômeno do estresse de cisalhamento depende da disponibilidade de L-arginina, levantou-se a possibilidade de que a suplementação com L-arginina poderia potencializar benefícios do exercício sobre a função endotelial. Sendo assim, o objetivo deste estudo foi investigar o efeito da suplementação de L-arginina sobre a resposta pressórica aguda, induzida por uma sessão de exercício em mulheres hipertensas de meia idade fisicamente ativas.

\section{MÉTODO}

\section{Amostra}

Considerando o tipo de estudo como experimental, adotou-se um procedimento estatístico para calcular o número do sujeito do estudo conforme a técnica do poder amostral. Para isto, adotou-se o software GPower 3.1.0. Com base em estudo prévio com população similar à do presente estudo (Palosshi et al., 2004), estimou-se uma diferença de PA sistólica de $20 \mathrm{mmHg}$, para um desvio-padrão residual de $12 \mathrm{mmHg}$. Adotando-se um poder estatístico de 0.80 e um nível de significância de 0.05 , foi estimado um $n$ mínimo de nove sujeitos para cada grupo estudado. O estudo foi desenvolvido com 20 mulheres portadoras de hipertensão de nível I e II de acordo com Joint National Committee on the Prevention, Detection, and Treatment of High Blood Pressure (JNC VI, 1997), de meia idade, previamente praticantes de exercícios aeróbios (caminhada) em um programa comunitário há pelo menos seis meses, com frequência de três vezes por semana. Todas eram usuárias de medicação anti-hipertensiva da classe dos inibidores da enzima conversora de angiotensina, e mantiveram a medicação durante todo o período do estudo. Elas foram distribuídas aleatoriamente em dois grupos: um grupo experimental (SUP), constituído por 10 mulheres que se submeteram ao uso de suplementação de L-arginina, e um grupo placebo (PLA) composto por 10 mulheres. A administração de L-arginina ou placebo foi feita seguindo o modelo duplo cego. Os procedimentos para este estudo foram tomados seguindo o que está estabelecido na declaração de Helsinque. O estudo foi previamente aprovado pelo comitê de ética em pesquisa do centro de ciências da saúde da Universidade Federal da Paraíba, sob o protocolo $n^{\circ}$ 038/08. Após aceitarem participar do estudo, todos os sujeitos assinaram o termo de consentimento livre e esclarecido. Em seguida, eles foram encaminhados para uma avaliação médica, como medida de segurança. Nessa avaliação, o cardiologista atestou estarem aptas à prática de exercícios físicos.

\section{Desenho do Estudo}

Os sujeitos do grupo SUP e PLA passaram um mês ingerindo um suplemento de L-arginina (3gr) e placebo respectivamente, duas vezes ao dia, sete dias por semana. Todas as mulheres continuaram com seus exercícios durante a fase experimental. Imediatamente antes e dois dias após o período de suplementação e placebo, elas realizaram uma sessão experimental de caminhada em esteira ergométrica. Frequência cardíaca e pressão arterial foram monitoradas antes, durante e após estas duas sessões de exercícios. Além disso, foram coletadas amostras sanguíneas de $8 \mathrm{ml}$, antes e 10 minutos após cada uma das duas sessões experimentais, para análise da concentração plasmática de nitrito/nitrato basal e em resposta ao exercício.

\section{Instrumentos e Procedimentos}

Análise de nitrito e nitrato séricos

As amostras de sangue foram colocadas 
em tubos de ensaio contendo EDTA e homogeneizadas suavemente por inversão. Em seguida, foi centrifugado a $1500 \mathrm{rpm}$ por 15 minutos e depois o plasma foi separado, colocado em tubos ependorf e congeladas a $80^{\circ} \mathrm{C}$ até a análise. A dosagem de nitrito/nitrato foi feita por meio de um kit comercial (Cayman Chemical Company, Michigan, USA), seguindo as recomendações do fabricante. Esse procedimento determina os níveis totais de óxido nítrico baseada na conversão enzimática de nitrato para nitrito pela enzima nitrato redutase. O plasma passou inicialmente por uma ultracentrifugação a uma velocidade de 10.000 rpm por 60 minutos, utilizando-se filtros de $30 \mathrm{kD}$ (Millipore, Bedford, MA, USA). A um volume de $40 \mu \mathrm{l}$ do ultrafiltrado foram adicionadas as seguintes substâncias fornecidas com o kit: $40 \mu$ de buffer, $10 \mu \mathrm{l}$ de um co-fator, $10 \mu \mathrm{l}$ de nitrato redutase, $50 \mu \mathrm{l}$ de reagente de Gries 1 e $50 \mu \mathrm{l}$ de reagente de Gries 2, totalizando $200 \mu$ l. O branco foi feito com $200 \mu$ de buffer, e uma curva padrão foi feita com diluições de 0 a $35 \mu \mathrm{l}$ de um padrão de nitrito/nitrato, a intervalos de $5 \mu \mathrm{l}$. A leitura foi feita em um leitor de placas ELISA, na faixa de absorbância de 542 $\mathrm{nm}$. As análises foram feitas em duplicata.

\section{Medição da Pressão Arterial (PA)}

Os sujeitos tiveram pressão arterial verificada antes, imediatamente após o término do exercício e durante os 30 minutos de encerrado o exercício. A medida de repouso foi verificada após os sujeitos permanecerem sentados por 10 minutos. Ao término da sessão de caminhada, a PA foi verificada com os sujeitos permanecendo sentados. Durante a recuperação, a PA foi verificada aos 10, 20 e 30 minutos de encerrado a sessão de caminhada. Adotou-se o protocolo proposto pelas Sociedade Brasileira de Cardiologia, Sociedade Brasileira de Hipertensão e Sociedade Brasileira de Nefrologia (2006).

\section{Exercício Físico}

Os grupos SUP e PLA foram submetidos a duas sessões de exercício aeróbio em esteira, sendo uma antes de iniciar o protocolo de suplementação ou placebo e a outra ocorreu 48 horas após este protocolo. As duas sessões tiveram um intervalo de 32 dias entre elas. O exercício teve duração de 40 minutos. A intensidade prescrita foi entre 50 e $60 \%$ da frequência cardíaca de reserva, sendo que a frequência cardíaca máxima foi estimada com base nas diretrizes recomendadas pelo American College of Sport Medicine (ACMS, 1998). Além disso, foi estabelecido que as mulheres deveriam se exercitar referindo pontuação entre 11 e 14 na escala de percepção subjetiva de esforço de Borg. Desta forma, foram feitas reduções na intensidade do exercício em relação ao que havia sido prescrito, baseado no método de prescrição do exercício pela frequência cardíaca apenas para as mulheres que referiram desconforto com a intensidade previamente prescrita. Como resultado, a intensidade realmente utilizada durante o protocolo de exercício ficou entre 40 a $60 \%$ da frequência cardíaca máxima. A velocidade da esteira foi anotada para que, na segunda sessão de exercício, a intensidade fosse controlada pela velocidade da esteira ergométrica realizada na primeira sessão.

\section{Suplementação de L-arginina}

A suplementação de L-arginina e o produto placebo foram produzidos em um laboratório de manipulação e passaram previamente por um exame de certificação (Pharma Nostra, João Pessoa, Brasil). O grupo SUP consumiu L-arginina, que foi acondicionado em saches contendo 3 gr de L-arginina e .03 gr. de aroma baunilha. O grupo placebo ingeriu um composto de .015 gr (.5\%) de aspartame, .015 gr $(.5 \%)$ de acesulfame, .03 gr (1\%) de aroma baunilha e $2.94 \mathrm{gr}$ de lactose. A pesquisadora e duas voluntárias fizeram visitas às participantes do estudo pela manhã e à tarde para realizar as entregas dos saches e verificar a ingestão de todo o conteúdo. Os sujeitos consumiram dois saches por dia, contendo $3 \mathrm{gr}$ 
cada, dissolvidos em água ou suco (Palloshi et al, 2004). O conteúdo dos saches foram dissolvidos e ingeridos na frente das pesquisadoras. Às sextas-feiras eram entregues os saches para o sábado e domingo, de modo que a ingestão nestes dias não foi supervisionada, mas após o fim de semana as participantes confirmavam a ingestão e entregavam os saches vazios.

\section{Determinação do consumo dietético de L-arginina}

Foi avaliado o consumo prévio e habitual de L-arginina a partir dos alimentos comumente ingeridos na dieta dos sujeitos do estudo. Para isto, uma profissional de nutrição aplicou um recordatório de 24 horas sugerido pela Dietary Recommendation Intake (DRI), conforme proposto por Fisberg, Slater, Marchioni, e Martini (2005), por ser o instrumento mais utilizado para este fim. Este método consiste em definir e quantificar todos os alimentos ingeridos no período de 24 horas anteriores à entrevista. $\mathrm{O}$ instrumento foi aplicado em três dias ao longo do procedimento de intervenção sendo um deles mensurando a dieta de um final de semana. Os dados foram avaliados pelo software Nutwin (Versão 1.5, 2003, Brasil). Em todas as entrevistas, as mulheres foram solicitadas a não alterar seus hábitos alimentares durante o período em que estariam sob uso da suplementação.

\section{Análise Estatística}

Os dados estão apresentados como média e desvio-padrão. Foi aplicado o teste de Smirnov-kolmogorov para testar a normalidade dos dados, seguido do teste Bartlett para determinar significância estatística entre os desvios-padrão dos dados que seriam posteriormente comparados. Considerando os resultados destes testes, foi utilizado o teste de ANOVA para medidas repetidas, com post hoc de Tukey, adotando nível e confiança de $95 \%$. Para comparar as características basais dos dois grupos, foi utilizado o teste $\mathrm{T}$ independente. Os testes foram rodados no software GraphPad
Instat, versão 3.0.6 (GraphPad Software inc, San Diego, CA, USA).

\section{RESULTADOS}

As mulheres do grupo SUP ou PLA apresentavam similaridade em idade, composição corporal e perfil lipídico sanguíneo. Os valores de base de frequência cardíaca e pressão arterial também eram similares antes do início do estudo. $\mathrm{O}$ inquérito nutricional revelou que $\mathrm{O}$ consumo de 1-arginina na dieta era adequado e sem diferenças entre os dois grupos. Estes dados estão apresentados na tabela 1 .

Tabela 1

Dados demográficos, antropométricos, clínicos e bioquímicos dos três grupos estudados.

\begin{tabular}{|c|c|c|}
\hline & $\begin{array}{l}\text { Experimental } \\
(n=10)\end{array}$ & $\begin{array}{l}\text { Placebo } \\
(n=10)\end{array}$ \\
\hline Idade (anos) & $50.0 \pm 1.8$ & $51.5 \pm 1.6$ \\
\hline $\operatorname{IMC}\left(\mathrm{Kg} / \mathrm{m}^{2}\right)$ & $26.8 \pm 1.0$ & $27.6 \pm 1.0$ \\
\hline $\begin{array}{l}\text { Circunferência } \\
\text { de cintura }(\mathrm{cm})\end{array}$ & $90.5 \pm 2.0$ & $91.0 \pm 2.0$ \\
\hline $\begin{array}{l}\text { Circunferência } \\
\text { do Quadril (cm) }\end{array}$ & $99.4 \pm 2.0$ & $101.9 \pm 1.0$ \\
\hline $\begin{array}{c}\text { Relação cintura } \\
\text { quadril }\end{array}$ & $0.9 \pm 0.02$ & $0.9 \pm 0.03$ \\
\hline $\begin{array}{l}\text { Colesterol } \\
\text { total (mg/dl) }\end{array}$ & $258.0 \pm 43.0$ & $190.0 \pm 18.0$ \\
\hline $\begin{array}{c}\text { Colesterol } \\
\text { LDL (mg/dl) }\end{array}$ & $118.0 \pm 38.0$ & $134.0 \pm 10.0$ \\
\hline FC (spm) & $72.6 \pm 4.0$ & $67.6 \pm 2.0$ \\
\hline PAS rep $(\mathrm{mmHg})$ & $137.0 \pm 4.0$ & $134.8 \pm 4.0$ \\
\hline PAD rep $(\mathrm{mmHg})$ & $87.2 \pm 2.0$ & $84.8 \pm 3.0$ \\
\hline $\begin{array}{c}\text { L-arginina } \\
\text { dietética }(\mu \mathrm{mol} / \mathrm{l})\end{array}$ & $4.2 \pm 0.5$ & $5.0 \pm 0.5$ \\
\hline
\end{tabular}

Valores são média e desvio-padrão da média. Não existe diferença estatística entre os dois grupos para nenhuma das variáveis; IMC = índice de massa corporal; FC = frequência cardíaca; PAS = pressão arterial sistólica; $\mathrm{PAD}=$ pressão arterial diastólica.

A intensidade dos exercícios realizados na sessão pré-intervenção ficou entre 40 e $60 \%$ da frequência cardíaca de reserva. Na sessão pós-intervenção, todas as mulheres adotaram a mesma velocidade na esteira que havia sido registrada no exercício anterior. 
Efeito da suplementação com L-arginina na concentração basal de nitrito / nitrato

$\mathrm{Na}$ análise pré-suplementação, os dois grupos apresentaram valores basais de nitrito / nitrato similares entre si. Após o período de intervenção, apenas o grupo SUP teve um aumento significativo na concentração sérica basal de nitrito / nitrato, a qual aumentou em $68 \%$ (Figura 1).

O exercício realizado não alterou a concentração de nitrito / nitrato em nenhum dos grupos estudados na condição pré-intervenção. Após o período de suplementação ou placebo, o grupo PLA continuou mostrando o mesmo comportamento do nitrito / nitrato em resposta ao exercício. Enquanto isso, o grupo SUP apresentou um aumento de $16 \%$ na concentração de nitrito/nitrato do repouso para o pós-exercício, mas com $p$ valor de .10 entre os valores pré e pós exercício. Os valores pré e pós-exercício do grupo SUP foram maiores que os do grupo PLA após a suplementação com L-arginina (Figura $1)$.

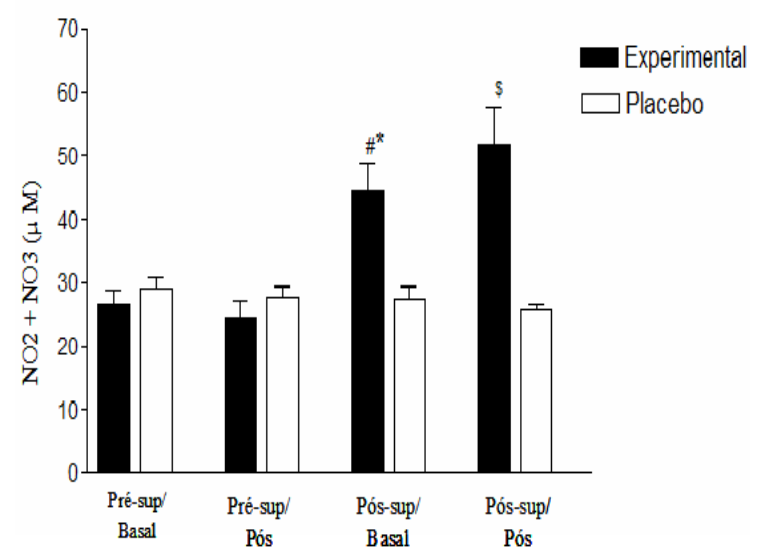

Figura 1. Níveis séricos de nitrito/nitrato basal e pós-exercício no período pré e pós suplementação

Efeito da suplementação de L-arginina na pressão arterial

Como resultado do protocolo de intervenção, o grupo SUP apresentou uma redução significativa da pressão arterial de repouso (de $137.0 \pm 4.0$ para $125.8 \pm 4.0$ para a pressão sistólica e de $86.2 \pm 2.0$ para $79.0 \pm 3.0$ para a pressão diastólica). No entanto, o exercício realizado no momento pós-suplementação não foi capaz de promover uma maior HPE em relação ao exercício realizado antes da suplementação de L-arginina neste grupo. Sendo assim, os grupos SUP e PLA apresentaram o mesmo comportamento da HPE, tanto antes quanto após o período de intervenção. As medidas de pressão arterial feitas aos 10, 20 e 30 minutos do período de recuperação pós-exercício mostraram que os menores valores de pressão foram obtidos sempre na última medida, confirmando que a HPE ocorre nos minutos mais tardios. Este fenômeno foi coincidente para os dois grupos e para os exercícios realizados antes e depois da intervenção com a suplementação. Estes dados estão sumariados na Figura 2A (pressão arterial sistólica), Figura 2B (pressão arterial diastólica) e Figura 3 (hipotensão pós exercício).
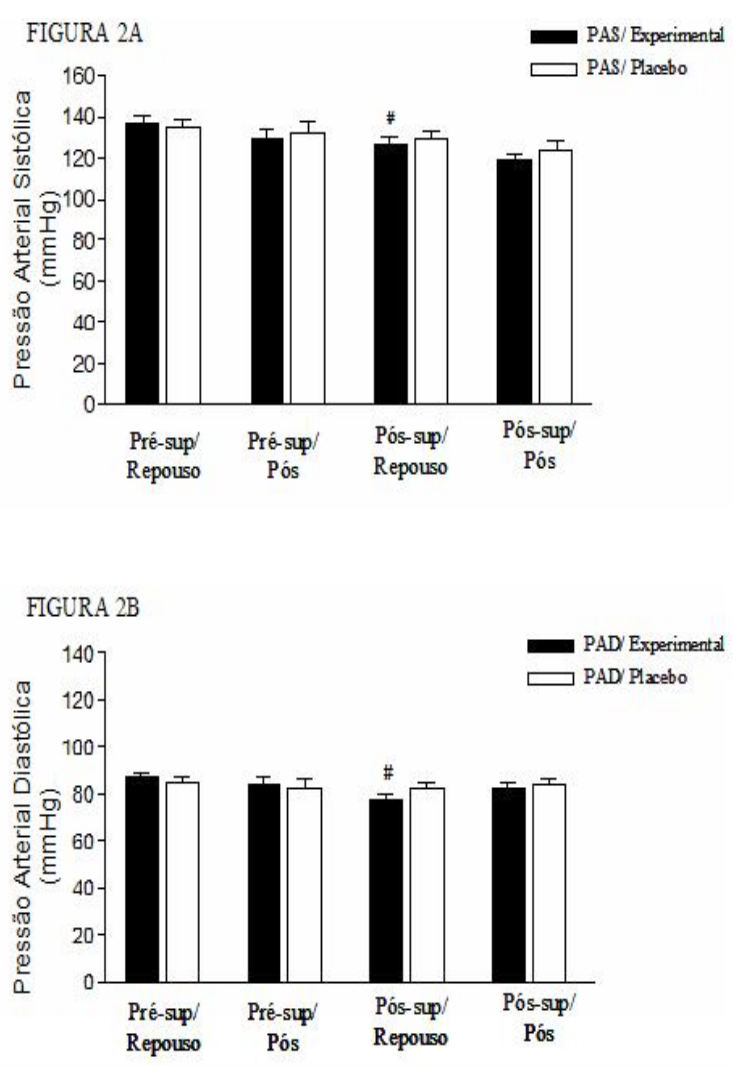

Figura 2. Valores da pressão arterial sistólica (PAS) e pressão arterial diastólica (PAD) de repouso, 30 
minutos após o exercício.

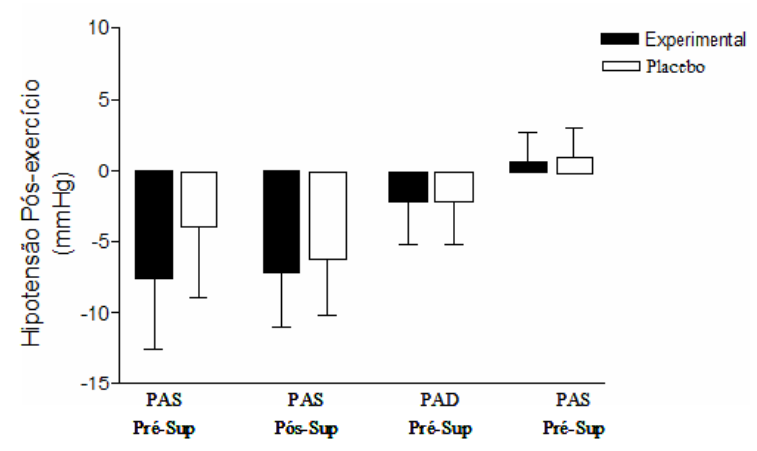

Figura 3. Hipotensão pós- exercício (HPE) nos momentos pré e pós-suplementação.

\section{DISCUSSÃO}

Os dados deste estudo confirmam a hipótese de que a suplementação com L-arginina reduz a pressão arterial de repouso e aumenta os níveis basais de nitrito/nitrato. Entretanto, refutam a hipótese de que esta melhoria da função endotelial e da pressão arterial poderia potencializar a hipotensão induzida por uma sessão de exercício.

Vários outros estudos com diferentes concentrações de L-arginina na suplementação, também mostraram aumento dos níveis de nitrito/nitrato (Lucotti et al., 2006; Martina et al., 2008; Rytlewski et al., 2005). Enquanto isso, algumas outras investigações falharam em demonstrar aumento na concentração plasmática e urinária de nitrito/nitrato em resposta à suplementação de L-arginina (Lim et al., 2004; Wilson, Harada, Nair, Balasubrananian, \& Cooke, 2007). Palloshi et al. (2004) usaram os mesmos 6 gr diários de suplementação do presente estudo. Eles encontraram melhoria da PA de repouso e do fluxo sanguíneo, mas o protocolo de suplementação também não afetou a concentração de nitrito/nitrato. Dentre estes estudos, os que mostraram efeitos foram realizados com sujeitos de meia idade ou idosos. Isto indica a possibilidade de que a condição prévia da função endotelial pode ser o fator que explica a diferença nestes resultados.

A redução da pressão arterial nos sujeitos suplementados encontrada neste estudo corrobora com uma variedade de investigações prévias (Lim et al., 2004; Martina et al., 2008; Paloshi et al., 2004; Rytlewski et al., 2005). Outro benefício da suplementação de L-arginina é o aumento do fluxo sanguíneo, o que também indica melhoria da função endotelial, diminuição do estresse oxidativo e inibição da agregação plaquetária, que colaboram para explicar a redução da pressão arterial (Adams et al., 1995; Lekakis et al., 2002; Lim et al., 2004). Por outro lado, alguns estudos têm mostrado redução da PA com suplementação de L-arginina, mesmo sem aumento da concentração plasmática e redução da concentração urinária de nitrito/nitrato (Hambrecht et al., 2000; Lim et al., 2004). A diminuição no estresse oxidativo, entretanto, pode resultar em maior biodisponibilidade de óxido nítrico, mesmo sem aumento de sua produção, uma vez que estas moléculas não mais agirão como antioxidante, passando a ficar disponíveis para ativação da via do GMPc no músculo liso, com consequente vasodilatação.

Apesar de o estresse de cisalhamento induzido pelo exercício ser um potente estímulo para a produção endotelial de óxido nítrico (Maeda et al., 2004), os dados do exercício pré-suplementação indicaram que as concentrações séricas de nitrito/nitrato não foram afetadas. Por outro lado, no exercício realizado após o período de suplementação com L-arginina, ocorreu um aumento de $16 \%$ na concentração plasmática de nitrito/nitrato da condição basal para o pós-exercício, embora este aumento tenha sido significativo. Fayh (2005) também não observou alteração significativa nos níveis plasmáticos de nitrito/nitrato para indivíduos diabéticos e controle após uma sessão de exercício aeróbio em cicloergômetro. Perez et al. (2002) demonstraram que ratos exercitados em baixas intensidades não apresentaram alterações na concentração de nitrito/ nitrato. Entretanto, para exercícios realizados em maior intensidade ocorreu aumento signi- 
ficativo na produção de NO. Sendo assim, estes dados explicam a ausência ou discreto aumento de nitrito/nitrato entre as mulheres do presente estudo, que realizaram o protocolo de exercício com intensidade leve para moderada.

Os mecanismos que podem explicar a redução ou a ausência de aumento do nitrito/ nitrato são as constatações de que nos exercícios de baixa intensidade, a pequena produção de nitrito/nitrato sofre um clearance ainda durante o exercício. $\mathrm{O}$ fato da coleta de sangue ter sido feita somente 10 minutos pós-exercício pode ter contribuído para este clearance. No entanto, a ausência de uma coleta feita imediatamente após o exercício impede a confirmação desta possibilidade. Perez et al. (2002) ainda propõem que a melhor capilarização induzida pelo exercício em sujeitos previamente treinados aprimora o clearance do nitrito/nitrato. Outra possível explicação para a ausência de aumento na produção de nitrito/nitrato seria o fato das mulheres terem melhorado seus níveis de condicionamento físico durante os 30 dias de intervenção do estudo. Entretanto, isto é muito pouco provável, porque elas já eram fisicamente ativas há pelo menos seis meses e não modificaram os protocolos de exercício durante o período do estudo.

Até onde sabemos, esta é a primeira vez que o efeito da suplementação de L-arginina na HPE foi investigado. A redução da pressão arterial, logo nos primeiros 20 a 30 minutos que sucedem uma única sessão de exercício, é um fenômeno bem evidenciado (Forjaz, Brandão-Rondon, \& Negrão, 2005). Os valores desta redução são considerados clinicamente importantes, porque são similares ao que são obtidos com a administração de uma das categorias de fármacos anti-hipertensivos e também porque a duração desta atividade hipotensora se aproxima de 24 horas, de modo que a realização diária de exercício físico mantém a pressão arterial cronicamente diminuída (Baster \& Baster-Brooks, 2005; Forjaz et al., 2005). Dentre os vários mecanismos propostos, alguns são rela- cionados à função neural (Irigoyen et al., 2003), enquanto outros são associados à função endotelial (Higashi \& Yoshizumi, 2004). O aumento da concentração plasmática basal de nitrito/ nitrato encontrado neste estudo reflete uma melhoria da função endotelial e pode explicar a importante redução da pressão arterial basal. Mas a ausência de aprimoramento da HPE indica que estas respostas basais não se transferem para a atividade endotelial durante o exercício.

\section{CONCLUSÕES}

Tomados em conjunto, os dados deste estudo mostram que a suplementação de 6 gr diárias de L-arginina por um período de quatro semanas é capaz de promover um aumento significativo nos níveis basais de nitrito e nitrato e reduzir a pressão arterial de repouso. Entretanto, não aprimora a hipotensão induzida por uma sessão de exercício físico aeróbio.

\section{Agradecimentos:}

Nada declarado.

Conflito de Interesses:

Nada declarado.

Financiamento:

Nada declarado.

\section{REFERÊNCIAS}

Accorsi-Mendonça, D., Almado, C. E. L., Fernandes, L. G., \& Machado, B. H. (2005). Controle neural da circulação e hipertensão arterial. Revista Brasileira de Hipertensão, 12 (4), 235-241.

American College of Sports Medicine Position Stand (1998). The recommended quantity and quality of exercise for developing and maintaining cardiorespiratory and muscular fitness, and flexibility in adults. Medicine and Science in Sports and Exercise, 30(6), 975-991.

Adams, M., Forsyth, C., Jessup, W., Robinson, 
J., \& Celermajer, D. (1995). Oral L arginine inhibits platelet aggregation but does not enhance endothelium-dependent dilation in healthy young men. Journal of the American College of Cardiology, 26(4), 1054-1061. doi: 10.1016/0735-1097(95)00257-9.

Araújo, C. G. S. (2001). Fisiologia do exercício físico e hipertensão arterial. Uma breve introdução. Revista Hipertensão, 4, 78-83.

Baster, T. \& Baster-Brooks, C. (2005). Exercise and hypertension. Australian Family Physician, 34(6), 419-424.

Boo, Y. C., \& Jo, H. (2003). Flow-dependent regulation of endothelial nitric oxide synthase: Role of protein kinases. American journal of physiology: Cell physiology, 285, 499-508. doi: 10,1152/ajpcell.00122.2003

Claudino, M. A., Priviero, F. B., Teixeira, C., De Nucci, G., Antunes, G., \& Zanesco. A (2004). Improvement in relaxation response in corpus cavernosun from trained rats. Urology, 63, 10041008. doi: 10.1016/j.urology.2003.11.034

Consolim-Colombo, F., \& Fiorino, P. (2005). Sistema nervoso simpático e hipertensão arterial sistêmica - aspectos clínicos. Revista Brasileira de Hipertensão, 12, 251-255.

Danson, E. J. \& Paterson, D. J. (2005). Cardiac neurobiology of nitric oxide synthases. Annals of the New York Academy of Sciences, 1047(1), 183-196. doi: 10.1196/annals.1341.017

Dequattro, V., \& Feng, M. (2002). The sympathetic nervous system: the muse of primary hypertension. Journal of Human Hypertension, 16, 64-69. doi: 10.1038/sj/jhh/1001346

Fayh, A. P. T. (2005). Efeito da suplementação de L-arginina e do exercício em cicloergômetro sobre a função endotelial e eestressee oxidativo em sujeitos com diabetes tipo I. MD Thesis, 2005. Universidade Federal do Rio Grande do Sul.

Fisberg, R. M., Slater, B., Marchioni, D. M. L., \& Martini, L. A. Inquéritos alimentares: métodos e bases científicos. Barueri, SP: Manole, 2005.

Forjaz, C. L. M., Brandão-Rondon, U. M., \& Negrão C. E. (2005). Efeitos hipotensores e simpatolíticos do exercício aeróbio na hipertensão arterial.
Revista Brasileira de Hipertensão, 12(4), 245-250.

Green, D. J., Maiorana, A., O'driscoll, G., \& Taylor, R. (2004). Effect of exercise training on endothelium-derived nitric oxide function in humans. The Journal of Physiology, 561, 1-25. doi: 10.1113/jphysiol.2004.068197

Griffin, K. L., Laughlin, M. H., \& Parker, J. L. (1999). Exercise training improves endothelium mediated vasorelaxation after chronic coronary occlusion. Journal of Applied Physiology, 87, 19481956.

Hambrecht, R., Hilbrich, L., Erbs, S., Gielen, S., Fiehn, E., Schoene, N., \& Schuler G. (2000). Correction of endothelial dysfunction in chronic heart failure: additional effects of exercise training and oral 1-arginine supplementation. Journal of the American College of Cardiology, 35, 706-713. doi: 10.1016/S0735-1097(99)00602-6

Higashi, Y., Oshima, T., Ozono, R., Matsuura, H., \& Kajiyama, G. (1997). Aging and severity of hypertension attenuate endotheliumdependent renal vascular relaxation in humans. Hypertension, 30, 252-258. doi: 10.1161/01. HYP.30.2.252

Higashi, Y. \& Yoshizumi, M. (2004). Exercise and endothelial function: Role of endotheliumderived nitric oxide and oxidative stress in healthy subjects and hypertensive patients. Pharmacology \& Therapeutics, 102(1), 87 - 96. http://dx.doi.org/10.1016/j. pharmthera.2004.02.003

Irigoyen, M. C., Lacchini, S., Angelis, K., \& Michelini, L. C. (2003). Fisiopatologia da hipertensão: O que avançamos? Revista da Sociedade de Cardiologia do Estado de São Paulo, 13, 20-45.

Jonh, S., \& Schmieder, R. E. (2003). Potential mechanisms of impaired endothelial function in arterial hypertension and hypercholesterolemia. Current Hypertension Reports, 5, 199-207. doi: 10.1007/s11906-003-0021-1

Joint National Committee on The Prevention, Detection, and Treatment of High Blood Pressure (1997). Sixth Report (JNC VI). Archives of Internal Medicine, 157, 2413-2446.

Kuru, O., Sentürk, U. K, Demir, N., Yesilkaya, A., 
Ergüler, G., \& Erkiliç, M. (2002). Effect of exercise on blood pressure in rats with chronic NOS inhibition. European Journal of Applied Physiology, 87(2), 134-140. doi: 10.1007/ s00421-002-0602-8

Lassègue, B., \& Clempus, R. (2003). Vascular $\mathrm{NAD}(\mathrm{P}) \mathrm{H}$ oxidases: Specific features, expression, and regulation. American Journal of Physiology - Regulatory, Integrative and Comparative Physiology, 285, 277-297. doi: 10.1152/ ajpregu.00758.2002

Lekakis, J. P, Papathanassiou, S., Papaioannou, T. G., Papamichael, C. M., Zakopoulos, N., Kotsis, V.,...Stamatelopoulos, S.F. (2002). Oral L-arginine improves endothelial dysfunction in patients with essential hypertension. International Journal of Cardiology, 86, 317-323. doi: 10.1016/S0167-5273(02)00413-8,

Lim, D. S., Mooradian, S. J., Goldberg, C. S., Gomez, C., Crowley, D. C, Rocchini, A. P., \& Charpie, J.R. (2004). Effect of oral L-arginine on oxidant estresse, endothelial dysfunction, and systemic arterial pressure in young cardiac transplant recipients. American Journal of Cardiology, 94, 828-831. doi: 10.1016/j.amjcard.2004.05.073

Lucotti, P., Setola, E., Monti, L. D., Galluccio, E., Costa, S., Sandoli, E. P.,...Piatti, P. (2006). Beneficial effects of a long-term oral L-arginine treatment added to a hypocaloric diet and exercise training program in obese, insulin resistant type 2 diabetic patients. American Journal of Physiology - Endocrinology and Metabolism, 291(5), 906-912. doi: 10.1152/ ajpendo.00002.2006

Maeda, S., Miyauchi, T., Kakiyama, T., Sugawara, J., Iemitsu, M., Irukayama-Tomobe, Y.,...Matsuda, M. (2001). Effects of exercise training of 8 weeks and detraining on plasma levels of endothelium-derived factors, endothelin-1 and nitric oxide, in healthy young humans. Life Sciences, 69(9), 1005-1016. doi: 10.1016/S00243205(01)01192-4

Maeda, S., Tanabe, T., Otsuki, T., Sugawara, J., Iemitsu, M., Miyauchi, T.,...Matsuda, M. (2004). Moderate Regular Exercise Increases Basal
Production of Nitric Oxide in Elderly Women. Hypertension Research, 27(12), 947-953. doi: 10.1291/hypres.27.947

Martina, V., Masha, A., Gigliardi, V. R., Brocato, L., Manzato, E., Berchior, A.,...Iannone, A. (2008). Long-Term N-Acetylcysteine and L-Arginine Administration Reduces Endothelial Activation and Systolic Blood Pressure in Hypertensive Patients With Type 2 Diabetes. Diabetes Care, 31(5), 940-944.

Moncada, S., Palmer, R. M., \& Higgs., E. A. (1991). Nitric oxide: Physiology pathophysiology and pharmacology. Pharmacological Reviews, 43, 109-142.

Moncada, S. (1997). Nitric oxide in the vasculature: Physiology and pathophysiology. Annals of the New York Academy of Sciences, 811, 60-67. doi: 10.1111/j.1749-6632.1997.tb51989.x

Palloshi, A., Fragasso, G., Piatti, P., Monti, L. D., Setola, E., Valsecchi, G.,....Morgonato, A. (2004). Effect of oral L-arginine on blood pressure and symptoms and endothelial function in patients with systemic hypertension, positive exercise tests, and normal coronary arteries. American journal of Cardiology, 93(7), 933-935. doi: 10.1016/j.amjcard.2003.12.040

Perez, A. C., Oliveira, C., Prieto, J. G., Ferrando A.,Vila, L., \& Alvarez, A. I. (2002). Quantitative assessment of nitric oxide in rat skeletal muscle and plasma after exercise. European Journal of Applied Physiology, 88, 189-191. doi 10.1007/ s00421-002-0693-2

Piccirilo, G., Munizzi, M. R., Fimognari, F. L., \& Marigliano, V. (1996). Heart rate variability in hypertensive subjects. International Journal of Cardiology, 53(3), 291-298. doi: 10.1016/01675273(95)02538-3

Rebelo, F. P. V., Benetti, M., Lemos L. S., \& Carvalho T. (2001). Efeito agudo do exercício físico aeróbio sobre a pressão arterial de hipertensos controlados submetidos a diferentes volumes de treinamento. Revista Brasileira de Atividade Física e Saúde, 6(2), 28-37.

Rytlewski, K., Olszanecki, R., Korbut, R., \& Zdebski, Z. (2005). Effects of prolonged 
oral supplementation with L-arginine on blood pressure and nitric oxide synthesis in preeclampsia. European Journal of Clinical Investigation, 35(1), 32-37. doi: 10.1111/j.13652362.2005.01445.x

Sarri, K., Linardakis, M., Codrington, C., \& Kafatos, A. (2007). Does the periodic vegetarianism of Greek Orthodox Christians benefit blood pressure? Preventive Medicine, 44(4), 341-348. doi: 10.1016/j.ypmed.2006.11.009

Sociedade Brasileira de Cardiologia, Sociedade Brasileira de Hipertensão, \& Sociedade Brasileira de Nefrologia. (2006). V Diretrizes Brasileiras de hipertensão arterial. Revista da Sociedade Brasileira de Hipertensão, 9(4), 256-312.

Sposito, A. C. (2004). Emerging insights into hypertension and dyslipidaemia synergies. European Heart Journal, 6, 8-12. doi: 10.1016/j. ehjsup.2004.10.003

Tatchum-Talom, R., Schulz, R., Mcneill, J. R., \& Khadour, F. H. (2000). Upregulation of neuronal nitric oxide synthase in skeletal muscle by swim training. American Journal of Physiology, 279, 1757-1766.
Touyz, R. M. (2005). Intracellular mechanisms involved in vascular remodeling of resistance arteries in hypertension: role of angiotensin II. Experimental Physiology, 90(4), 449-455. doi: 10.1113/expphysiol.2005.030080

Vanhoutte, P. M. (2003). Endothelial control of vasomotor function: From health to coronary disease. Circulation Journal, 67(7), 572-575. doi: 10.1253 / circj. 67.572

Wang, J. S. (2005). Effects of exercise training and detraining on cutaneous microvascular function in man: The regulatory role of endotheliumdependent dilation in skin vasculature. European Journal of Applied Physiology, 93 (4), 429-434. doi: 10.1007/s00421-004-1176-4

Wilson, A. M., Harada, R., Nair, N., Balasubramanian, N., \& Cooke, J. P. (2007). L-Arginine Supplementation in Peripheral Arterial Disease. No Benefit and Possible Harm. Circulation Journal, 116(2), 188-195. doi: 10.1161/circulationaha.106.683656

\section{(cc) BY-NC} Todo o conteúdo da revista Motricidade está licenciado sob a Creative Commons, exceto quando especificado em contrário e nos conteúdos retirados de outras fontes bibliográficas. 